Featured Letter

\title{
Designing biomaterials for tissue engineering based on the deconstruction of the native cellular environment
}

\author{
João F. Mano ${ }^{\mathrm{a}, \mathrm{b}, *}$ \\ a 3B's Research Group - Biomaterials, Biodegradables and Biomimetics, University of Minho, AvePark, Zona Industrial da Granja, S. Cláudio do Barco, \\ 4806-909 Caldas das Taipas, Portugal \\ b ICVS/3B's - PT Government Associate Laboratory, Braga/Guimarães, Portugal
}

\section{A R T I C L E I N F O}

\section{Article history:}

Received 24 July 2014

Accepted 15 November 2014

Available online 25 November 2014

Keywords:

Biomaterials

Processing

Biomimetics

Stem cell niche

Regenerative medicine

Nanobiomaterials

\begin{abstract}
A B S T R A C T
Strategies in Tissue Engineering and Regenerative Medicine are often based on the use of biomaterials able to support and control cellular activity. Two aspects should be considered in the development of high performance bioinstructive biomaterials. (i) The inherent complexity associated with the multiple possibilities in the biomaterials/cells selection, usually addressed using high-throughput combinatorial tests; and (ii) the unpredictability of the biological outcome of a particular solution. The last facet requires a rational decomposition of the main spatial and temporal cues at the cellular level that drive new-tissue formation upon injury, to be then transposed into adequate biomaterials' design. Several nano/micro-technologies may be used to process biomaterials with different shapes and sizes, permitting to engineer biomimetic and hierarchical biomedical devices. As a particular case study, the layer-by-layer assembly method is suggested as a versatile and robust framework to formulate multifunctional and tunable polymer-based biomaterials able to address this exercise of deconstruction and reconstruction.
\end{abstract}

(c) 2014 Elsevier B.V. All rights reserved.

\section{Introduction}

Key strategies in regenerative medicine rely on the combination of cells with a biodegradable matrix that could support the right cellular activity by emulating central molecular and structural features of the extracellular matrix (ECM). Biodegradable biomaterials have been recognised for a long time to have a pivotal role in the field of tissue engineering (TE), as they could act as temporary biomimetic supports to maintain cell viability and control cellular behaviour [1,2]. To provide the right signals to cells, biomaterials should carry complex information coded in their physical and chemical structures [3]. Although important generic relationships between biomaterials characteristic and the corresponding biological outcome have been already established, such correlative process is still quite empirical and unpredictable. As it will be explored further, a critical intellectual step in biomaterials development for TE is to recognise: (i) the inherent convolution of the multi-variables that can feed the design of such devices (complexity) and; (ii) the absolute need to test any promising solution using adequate models to assess its therapeutic potential (unpredictability).

\footnotetext{
* Corresponding author at: 3B's Research Group - Biomaterials, Biodegradables and Biomimetics, University of Minho, AvePark, Zona Industrial da Granja,

S. Cláudio do Barco, 4806-909 Caldas das Taipas, Portugal.

E-mail address: jmano@dep.uminho.pt
}

Traditionally, biomaterials development for TE has been based on trial and error assessments, during which relevant clues could be empirically hinted on how architecture and physicochemical properties of devices could influence the performance of different cell types for example: the correlation between wettability and both protein adsorption and cell fate [4]; or the importance of pore interconnectivity and pore size in cell colonisation in 3D scaffolds [5]. Consistent progression in the field should include methodologies that could rationalise both complexity and unpredictability in biomaterials research. Two distinct methodologies could be considered.

The first one relies on the development of high-throughput methodologies to establish quantitative correlations between a great number of distinct materials and the corresponding biological behaviour [6,7]. High-throughput screening could be seen as an extension of the low-content analysis that has been recurrently used. From the miniaturisation of biomaterials' structures and the employment of adequate characterisation tools it has been possible to analyse massive combinations of materials, by exploring parameters such as macromolecular chemistry, wettability, topography, or mechanical properties. Multiple solutions of organising biomaterials to be tested have been proposed for such analysis, usually in the form of arrays or gradients. We developed a platform based on superhydrophobic substrates exhibiting patterned wettable spots to support 3D biomaterials for both in-vitro [8,9] and in-vivo [10] high-throughput screening. High-throughput methodologies permit 
to work under reduced a priori assumptions because any possible combination will be considered as equally valid as the others before the testing. Using adequate statistical tools to analyse the data it is possible to infer on general trends and cross-dependencies between variables, that could be useful in the development of new biomaterials [11].

Another approach is to start with some initial presumptions especially derived from fundamental evidences on how cells interact with the native milieu, in particular during tissue formation or remodelling. In this context it is relevant to understand how cells are embedded and function in this specialised complex environment, also called niches, that encompass ECM organisation at different length scales and involves multi-cellular spatio-temporal signalling processes [12]. The full picture of how this choreography takes place is far from being understood. However there are a variety of specific elements that revealed recognised importance and that could be decoupled to be investigated in a more modular and rationale form. This Feature Letter intends to recapitulate the major aspects that worth to be deconstructed from the convoluted niche and be explored towards the engineering of biomimetic regenerative environments.

The organisation of this Letter starts by exposing the most relevant factors that should be considered in biomaterials design, evidencing the fact that this exercise should go beyond the simple reproduction of the structure of native tissues. Then a section reflects on generic considerations concerning the choice of biomaterials and their processing at different scales and dimensional levels. Finally a specific section highlights the particular possibility of using layer-by-layer assembly as a fabrication toolbox of building blocks that could transpose the design principles into devices for TE.

\section{Relevant factors in the design of biomaterials}

Tissues and organs are complex and hierarchically organised (see scheme in Fig. 1A). Artificial systems cannot reproduce this complex structure. Nevertheless many general physiological functions and characteristics have been replicated in order to develop implantable biomaterials for replacement strategy proposes. Historical examples are orthopaedic implants with mechanical properties matching those of bone [13] or biomaterials for cardiovascular application with non-fouling interfacial properties [14]. In TE, biomaterials constitute an in-vitro alternative of the ECM, but in this case the design of such three-dimensional template does not need to match compulsory the entire structural organsation of the native tissue; it should rather provide the structural and instructive framework over which seeded or recruited cells can build by themselves the final functional tissue. For example, in native tissues cells are tightly entrenched within a dense macromolecular environment. Strategies using porous scaffolds should opt to another internal architecture for cell colonisation as it is well know that such structures should exhibit interconnected pores with sizes considerably higher than those of cells [5]. Other practical aspects should be considered in biomaterials design that are not observable in real tissues. For example, stimuli-responsive biomaterials may be used in TE [15], in order to produce injectable systems, substrates able to control cell attachment depending on temperature, or even scaffolds with shape memory triggered by hydration [16]. Moreover, it is known that ECM is continuously remodelling over time, but in synthetic counterparts the overall degradation of the biomaterial follows typically another dynamics, controlled mainly by the balance between the loss of mechanical properties and the increase of robustness due to new tissue formation [17].

Deciphering the role of particular topographic, physical and chemical/biochemical facets taking place in a cell's niche has emerged as a modern paradigm to develop biomaterials aiming regenerating tissues. Fig. 1B present some of such important features, recognised by many authors [18,19], and how they could be reproduced in biomaterials and culturing conditions. The deconstruction of these essential biophysical and structural elements can be reproduced synthetically by the correct design of biomaterialsbased scaffolds that could be then used as frameworks for simplified in vitro niches. Compromises should be assumed as many distinct elements are correlated and their disconnection may lead to the loss of bioactive effects. Moreover, the main difficulty of such approach is to know how that piecewise information obtained from the studies of the different deconstructed elements could be gathered in order maximise the bio-instructive potential of a device. A possible way to overcome this problem is to proceed with partial "reconstructions" of the previously analysed elements (i.e. the rationale assembly/integration of relevant biomaterials' elements into a macroscopic device) and make new in vitro tests, which could be also supported by high-throughput analysis. The last section highlights that important biomaterials components that could help in cell attachment and proliferation could be organised ("reconstruction" step) using layer-by-layer assembly.

\section{Formulating biomaterials for tissue engineering}

The fundamental information discussed in the last section should be then integrated in the pipeline of biomaterials' preparation that generically includes three steps: (i) synthesis/compounding, (ii) fabrication/assembling and (iii) modification. Researchers have usually sought assistance from chemistry to deal with the first topic. In the last 20 years strong efforts have been devoted to the preparation and use of different macromolecules, either synthetic [20] or natural-based [21], as structural building blocks in TE, often based on biomaterials previously proposed for other biomedical applications. Especially for bone TE, polymers have been also combined with inorganic elements, such as bioactive nanoparticles [22], that could be seen as a simple deconstruction of the composite nature of bone tissue. New biomaterials have been designed specifically for TE, by including, for example, the essential bioactive molecules found in the ECM using different strategies: by compounding native ECM proteins in the formulation; by introducing relevant aminoacids sequences in synthetic polymers through recombinant technologies or; by designing and combining with macromolecules simplified synthetic mimics able to reproduce the functional elements of ECM molecules [3].

Besides chemistry, physical aspects related to shape and size should be also considered in the fabrication or assembling processes [23]. The latest developments in the field of nano/micro-technologies enable processing polymers with a variety of shapes and covering all length-scales - see Fig. 2. "0-dimensional" structures (Fig. 2A-C), as a simplistic extrapolation of points, can be considered as spherical objects, including capsules and solid particles, that have been used in TE as delivery systems of bioactive agents or as structures or building-blocks to support cell attachment [24]. By far, the most usual "1-dimensional" nano-structures (Fig. 2D) are the fibres prepared by electrospinning, widely used as building blocks to fabricate artificial ECM matrices for TE [25]. Microfluidics are used to produce versatile micro-sized fibres [26] (Fig. 2E) and, for example, wetspinning permits to process fibres with larger calibre [27] (Fig. 2F). For the case of two-dimensional nanostructures thin or multilayered nanostructured (see next section) coatings or films have been proposed (Fig. 2G). 2D systems have been also produced with micro-size thicknesses or as surfaces with well-defined topographies or patterns [28] (Fig. 2H). 2D elements can be geometrically rearranged to present higher scale structures (Fig. 2I). Self-assembled nanostructures are obtained either using low-molecular weight molecules or macromolecules, and can be used to encapsulate cells or as instructive three-dimensional biomaterials [29] (Fig. 2J). Microsized hydrogel building blocks may be assembled permitting 


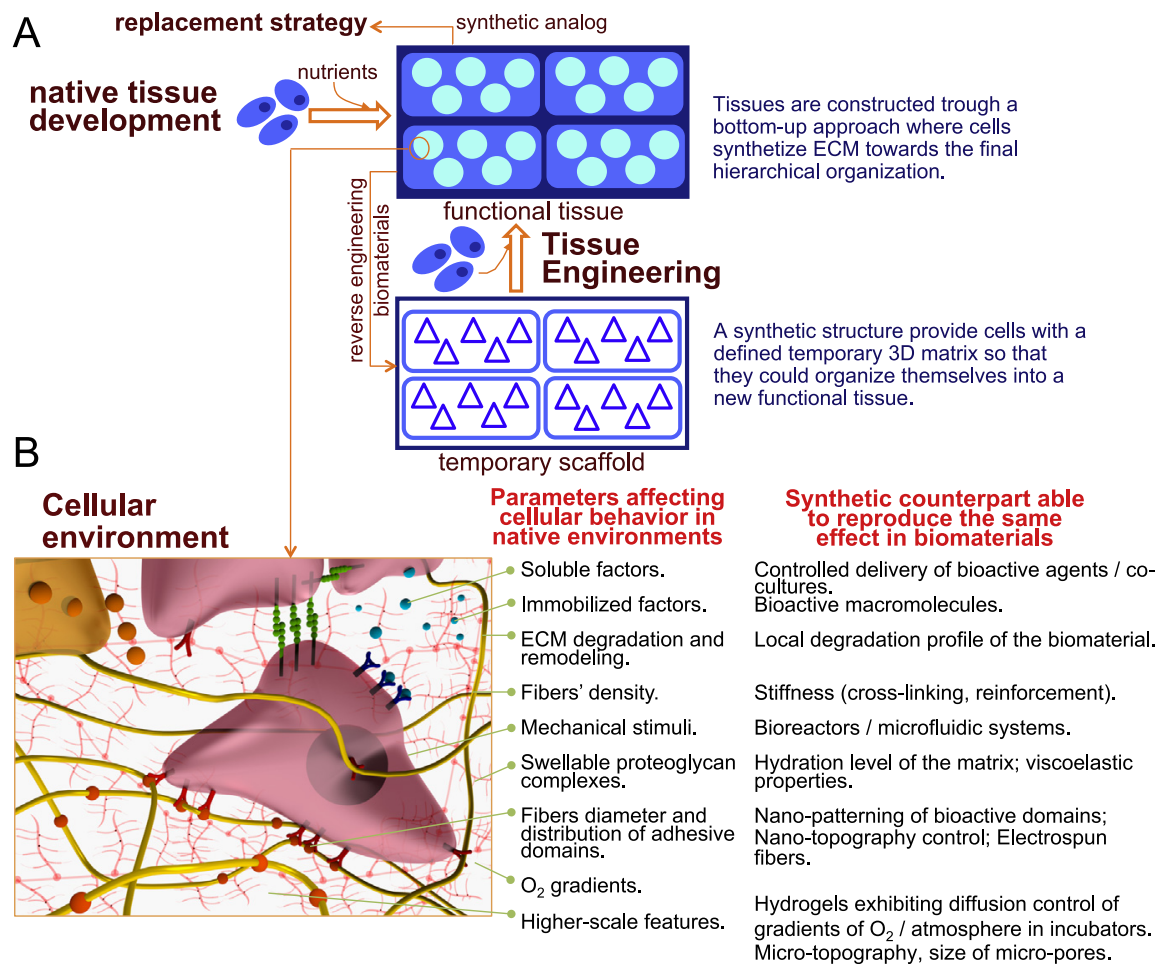

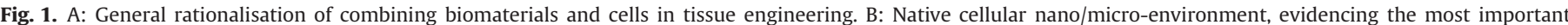
factors affecting cellular behaviour and the corresponding strategies to implement them through biomaterials' design.

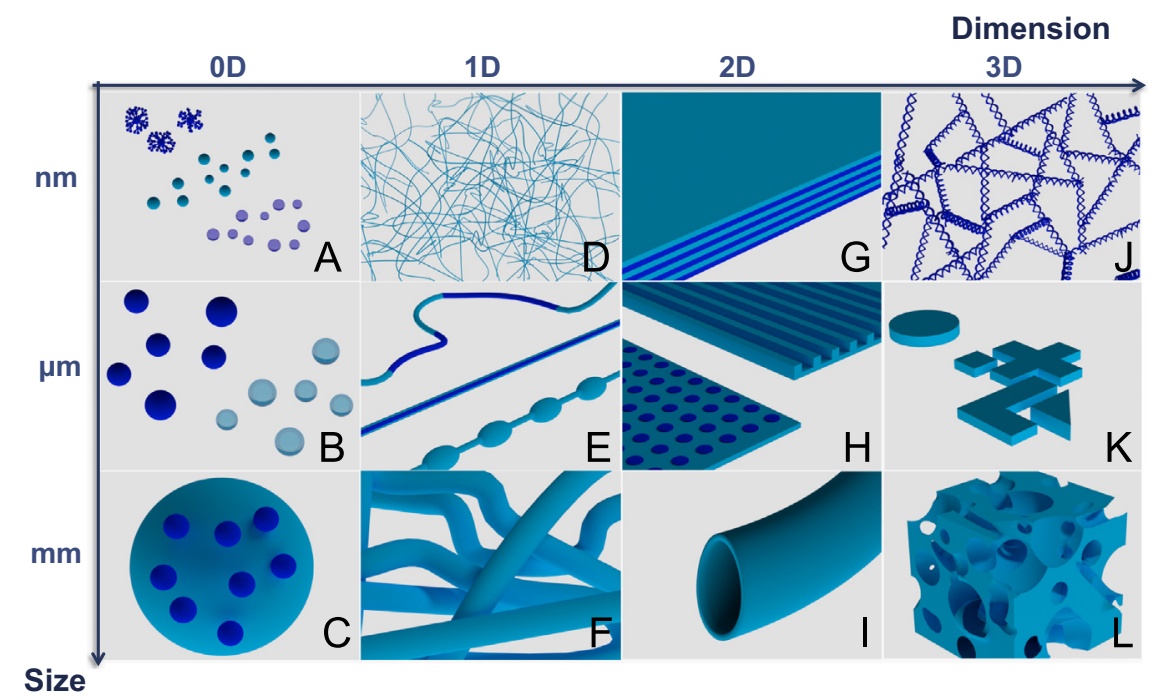

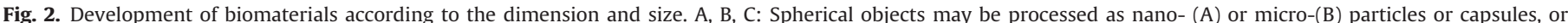

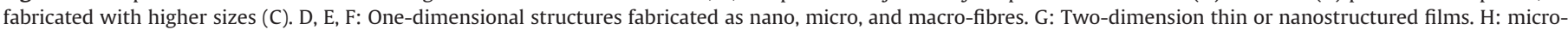

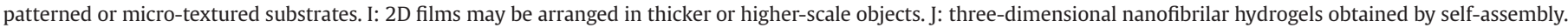
$\mathrm{K}$ : micro-scale tissues used as building blocks to produce complex structures. L: traditional porous scaffolds.

obtaining larger and more complex structures (Fig. 2K) using bottomup strategies [30]. Macro-scale porous structures or hydrogels (Fig. 2L) may be directly produced using a variety of techniques, constituting the most common way to produce devices for TE.

Elements shown in Fig. 2 may be combined across lengthscales or dimension. As an example of traversing the vertical axis, magnetic nanoparticles were incorporated into microcapsules that were then integrated into $\mathrm{mm}$-sized liquefied capsules coated with natural-based polyelectrolytes, in order to prepared compartmentalised multi-functional hierarchical structures [31]. Another example, now crossing the horizontal axis of Fig. 2, is the use of bioactive nanoparticles (OD) that were incorporated into electrospun nanofibers (1D) to produce thin non-woven films (2D) for bone TE [32]. Those are representative illustrations on how modern technologies, for example using thermal and solvent-based methodologies, may be use to process biomaterials that could capture relevant elements to meet specific practical specifications for a given TE strategy. In the next section a particular fabrication methodology will be highlighted.

Relevant aspects of the stem cell niche are presented in Fig. 1B, that have inspired researchers to develop biomimetic models for TE [12]. It is important to emphasise that the steady scenario of healthy tissues is not the ideal model to be reproduced when we intend to stimulate regeneration and new tissue formation. Lessons can be taken from, at least, two distinct situations: (i) during injury/trauma, 

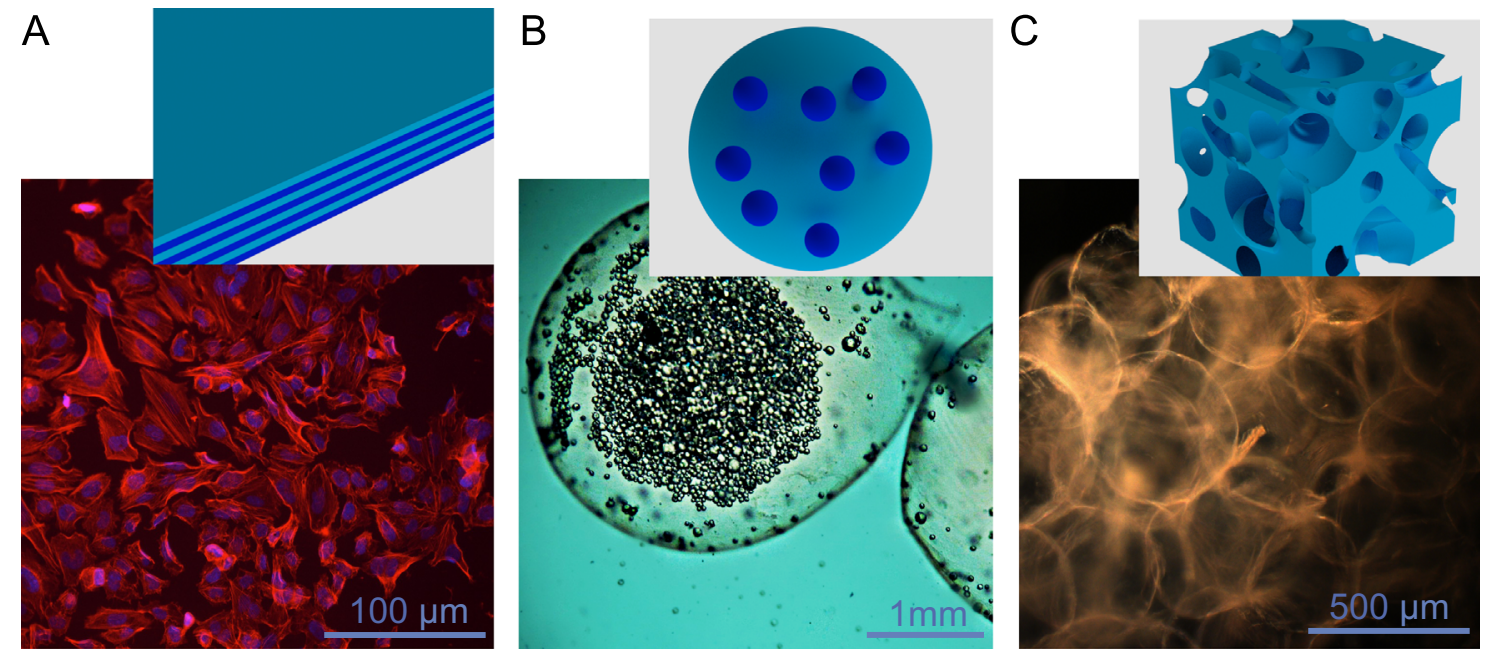

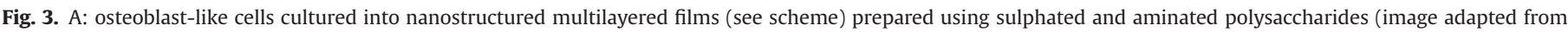

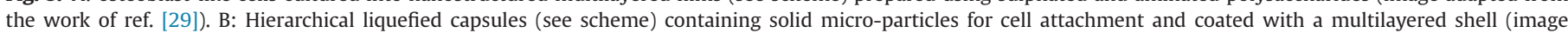

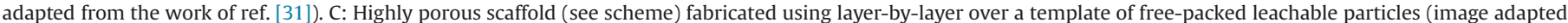
from the work of ref. [32]).

the niche loose his basal equilibrium and cells are exposed to special complex spatio-temporal biochemical signals to direct migration, proliferation and differentiation towards local healing or (ii) during embryonic development, where complex and vascularised tissues and organs are formed from potent biological precursors. It is of major importance to understand the specific factors that drives the two abovementioned processes. Among these factors, an important aspect is to control stem cell differentiation, that has been traditionally focused on the delivery of soluble bioactive morphogens. However, much more studies will be needed to instruct biomaterials not only to support normal cellular activity but also to stimulate a true regenerative process. Of special significance will be the collaborative efforts that engineers should undertake together with stem cell and developmental biologists.

\section{Layer-by-layer to produce controllable constructions for TE}

Many technologies have been used to transpose biomaterials design principles into devices for TE. In this section the particular case of layer-by-layer ( $\mathrm{LbL}$ ) assembly is presented as a versatile tool to fabricate temporary matrices with tuned structural and functional features. LbL is based on the sequential adsorption of at least two distinct materials onto substrates. Usually electrostatic forces have been the major interactions explored in the assembling process, but many other others could be used, including hydrogen bonds, hydrophobic interactions, covalent bonds or host-guest interactions [33]. LbL assembly is a simple, inexpensive, efficient, and highly versatile method to modify surfaces, including non-flat substrates, and fabricate nanostructured (natural or synthetic) polymeric thin films or nanocomposites with tailored structural and functional properties. In the last two decades, this technique has been used to produce substrates for a variety of biomedical applications, in particular for TE [34]. Most of the objects shown in Fig. 2 could be produced using (or integrating) this technology. Three representative examples from our group are shown in Fig. 3, that highlight the potential of multilayered-based biomaterials in deconstructing important aspects from native cellular environments:

Fig. 3A - Multilayers containing combinations of distinct carrageenans (bearing different densities of sulphate groups), chitosan and alginate were developed to prepare highly organised nanostructured films exposing distinct relative amounts of $-\mathrm{COOH},-\mathrm{NH}_{2}$ and $-\mathrm{SO}_{3} \mathrm{H}$ groups, that also exist in the proteoglycans-fraction of the ECM. Depending on the assembled biopolymers, cultured osteoblastlike cells could exhibit distinctive behaviour, including different biomineralisation ability [35].

Fig. 3B - Liquified capsules, protected by a shell produced using LbL, are able to compartmentalise adherent cells by including in the interior solid microparticles to provide surface anchorage points for cells $[36,37]$. The system can act as a true microbioreactor, offering a controlled microenvironment separated by a permselective frontier with the outside milieu.

Fig. 3C - Highly porous scaffolds formed entirely with polymeric multilayers offer well-regulated biointerfaces to control cellular behaviour $[38,39]$. Hierarchical porous devices have been also suggested by integrating nano/micro-fibrilar structures generated using LbL into the pores of pre-processed scaffolds [40].

It is clear from these examples that LbL permits achieving high degrees of functionalisation and geometrical complexity. Moreover, the possibility of fabricating thin films with nanometric scale control over their internal structure turns LbL as a suitable instrument to deconstruct many particular features of the cell niche programmed for regeneration. Some aspects normally associated with the native cellular microenvironment will be enumerated, and (using the symbol " $<=>$ ") confronted with characteristics connected with the LbL processing method:

- The ECM is formed through self-assembly with minimal energy input $<=>$ in LbL also bottom-up assembling is employed under mild (aqueous-based) conditions and involving weak molecular interactions.

- Cellular environment is hierarchically organised $<=>$ LbL permits to produce nanostratified building block elements that can be assembled into larger-scale structures. As different molecular arrangements may be found within the layers, it is possible to assume that this technique permits to have structural control from the molecular up to the macro-scale level.

- The niche is "information-rich" $<=>$ multilayers may be produced using a variety of biomaterials (including polymers and inorganic nano-objects) that can be disposed at will during its construction and may be used as reservoirs of distinct bioactive molecules. Moreover, post-processing can be done, such as cross-linking or surface chemical modifications. 
- Depending on the specific tissue, the ECM is normally produced in a reproducible manner $<=>$ by controlling parameters such as $\mathrm{pH}$, polyelectrolytes concentration and ionic strength multilayers may be built-up with precise and reproducible nano-architecture and thickness.

\section{Conclusions}

Knowledge from the deconstruction of relevant elements associated with the organisation and functioning of the native cellular microenvironment can be transposed to support the design of synthetic polymer-based elements for the fabrication of biomaterials' devices to be used in tissue engineering. Biomaterials should be processed into suitable structures, which do not need necessarily to be the conventional porous three-dimensional scaffolds. It was shown that layer-by-layer constitutes an adequate framework to engineer biomimetic regenerative environments, starting from the assembly of nano-organised multilayers.

\section{Acknowledgements}

The author is grateful for the Portuguese Foundation for Science and Technology (FCT) financial support in the scope of project PTDC/ CTM-BIO/1814/2012. The author acknowledges Dr. Iva Pashkuleva for the critical review of the manuscript and Dr. Luca Gasperini for preparation of several schemes.

\section{References}

[1] Hubbell JA. Biomaterials in tissue engineering. Nat Biotechnol 1995;13:565-76.

[2] Shin H, Jo S, Mikos AG. Biomimetic materials for tissue engineering. Biomaterials 2003;24(24):4353-64.

[3] Place ES, Evans ND, Stevens MM. Complexity in biomaterials for tissue engineering. Nat Mater 2009;8(6):457-70.

[4] Song W, Mano JF. Interactions between cells or proteins and surfaces exhibiting extreme wettabilities. Soft Matter 2013;9(11):2985-99.

[5] Karageorgiou V, Kaplan D. Porosity of 3D biomaterial scaffolds and osteogenesis. Biomaterials 2005;26(27):5474-91.

[6] Peters A, Brey DM, Burdick JA. High-throughput and combinatorial technologies for tissue engineering applications. Tissue Eng Part B 2009;15(3):225-39.

[7] Oliveira MB, Mano JF. High-throughput screening for integrative biomaterials design: exploring advances and new trends. Trends 2014;32(12):627-36.

[8] Salgado CL, Oliveira MB, Mano JF. Combinatorial cell-3D biomaterials cytocompatibility screening for tissue engineering using bioinspired superhydrophobic substrates. Integrat Biol 2012;4(3):318-27.

[9] Oliveira MB, Salgado CL, Song WL, Mano JF. Combinatorial on-chip study of miniaturized 3D porous scaffolds using a patterned superhydrophobic platform. Small 2013;9(5):768-78.

[10] Oliveira MB, Ribeiro MP, Miguel SP, Neto AI, Coutinho P, Correia IJ, et al. In vivo evaluation of three-dimensional scaffolds biocompatibility. Tissue Eng Part C 2014;20(11):851-64.

[11] Oliveira MB, Luz GM, Mano JF. A combinatorial study of nanocomposite hydrogels: on-chip mechanical/viscoelastic and pre-osteoblast interaction characterization. J Mater Chem B 2014;2(34):5627-38.

[12] Forbes SJF, Rosenthal N. Preparing the ground for tissue regeneration: from mechanism to therapy. Nat Med 2014;20(8):857-69.
[13] Mano JF, Sousa RA, Boesel LF, Neves NM, Bioinert Reis RL. Biodegradable and injectable polymeric matrix composites for hard tissue replacement: state of the art and recent developments. Compos Sci Technol 2004;64(6):789-817.

[14] Deible CR, Petrosko P, Johnson PC, Beckman EJ, Russell AJ, Wagner WR. Molecular barriers to biomaterial thrombosis by modification of surface proteins with polyethylene glycol. Biomaterials 1998;19(20):1885-93.

[15] Mano JF. Stimuli-responsive polymeric systems for biomedical applications. Adv Eng Mater 2008;10(6):515-27.

[16] Correia CO, Mano JF. Chitosan scaffolds with a shape memory effect induced by hydration. J Mater Chem B 2014;2(21):3315-23.

[17] Hutmacher DW. Scaffolds in tissue engineering bone and cartilage. Biomaterials 2000;21(24):2529-43.

[18] Hazeltine LB, Selekman JA, Palecek SP. Engineering the human pluripotent stem cell microenvironment to direct cell fate. Biotech Adv 2013;31(7):1002-19.

[19] Das RK, Zouani OF. A review of the effects of the cell environment physicochemical nanoarchitecture on stem cell commitment. Biomaterials 2014:35(20):5278-93.

[20] Gunatillake PA, Adhikari R. Biodegradable synthetic polymers for tissue engineering. Eur Cell Mater 2003;5:1-16.

[21] Mano JF, Silva GA, Azevedo HS, Malafaya PB, Sousa RA, Silva SS, et al. Natural origin biodegradable systems in tissue engineering and regenerative medicine: present status and some moving trends. J R Soc Interf 2007;4(17):999-1030.

[22] Boccaccini AR, Erol M, Stark WJ, Mohn D, Hong ZK, Mano JF. Polymer/bioactive glass nanocomposites for biomedical applications: a review. Comp Sci Tech 2010;70(13):1764-76.

[23] Mitragotri S, Lahann J. Physical approaches to biomaterial design. Nat Mater 2009;8(1):15-23.

[24] Oliveira MB, Mano JF. Polymer-based microparticles in tissue engineering and regenerative medicine. Biotechnol Progr 2011;27(4):897-912.

[25] Sill TJ, von Recum HA. Electro spinning: applications in drug delivery and tissue engineering. Biomaterials 2008;29(13):1989-2006.

[26] Kang E, Jeong GS, Choi YY, Lee KL, Khademhosseini A, Lee SH. Digitally tunable physicochemical coding of material composition and topography in continuous microfibers. Nat. Mater 2011;10(11):877-83.

[27] Malheiro VN, Caridade SG, Alves NM, Mano JF. New poly(epsilon-caprolactone)/chitosan blend fibers for tissue engineering applications. Acta Biomater 2010;6(2):418-28.

[28] Alves NM, Pashkuleva I, Reis RL, Mano JF. Controlling cell behavior through the design of polymer surfaces. Small 2010;6(20):2208-20.

[29] Aida T, Meijer EW, Stupp SI. Functional supramolecular polymers. Science 2012;335(6070):813-7.

[30] Elbert DL. Bottom-up tissue engineering. Curr Opin Biotech 2011;22(5):674-80.

[31] Costa RR, Castro E, Javier Arias F, Rodriguez-Cabello JC, Mano JF. Multifunctional compartmentalized capsules with a hierarchical organization from the nano to the macro scales. Biomacromolecules 2013;14(7):2403-10.

[32] Silva CSR, Luz GM, Gamboa-Martinez TC, Mano JF, Ribelles JLG, Gomez-Tejedor JA. Poly( $\varepsilon$-caprolactone) electrospun scaffolds filled with nanoparticles. Production and optimization according to taguchi's methodology. J Macromol Sci B 2014;53(5):781-99.

[33] Borges J, Mano JF. Molecular interactions driving the layer-by-layer assembly of multilayers: a review. Chem Rev 2014;114(18):8883-942.

[34] Costa RR, Mano JF. Polyelectrolyte multilayered assemblies in biomedical technologies. Chem Soc Rev 2014;43(10):3453-79.

[35] Oliveira SM, Silva TH, Reis RL, Mano JF. Nanocoatings containing sulfated polysaccharides prepared by layer-by-layer assembly as models to study cellmaterial interactions. J Mater Chem B 2013;1(35):4406-18.

[36] Correia CR, Sher P, Reis RL, Mano JF. Liquified chitosan-alginate multilayer capsules incorporating poly(L-lactic acid) microparticles as cell carriers. Soft Matter 2013;9(7):2125-30.

[37] Correia CR, Reis RL, Mano JF. Multilayered hierarchical capsules providing cell adhesion sites. Biomacromolecules 2013;14(3):743-51.

[38] Sher P, Custodio CA, Mano JF. Layer-by-layer technique for producing porous nanostructured 3D constructs using moldable freeform assembly of spherical templates. Small 2010;6(23):2644-8.

[39] Silva JM, Georgi N, Costa R, Sher P, Reis RL, Van Blitterswijk CA, et al. Nanostructured 3D constructs based on chitosan and chondroitin sulphate multilayers for cartilage tissue engineering. PloS One 2013;8(2):e55451.

[40] Oliveira SM, Silva TH, Reis RL, Mano JF. Hierarchical fibrillar scaffolds obtained by non-conventional layer-by-layer electrostatic self-assembly. Adv Health. Mater 2013;2(3):422-7. 